\title{
LOCALLY $e$-FINE MEASURABLE SPACES
}

$$
\text { BY }
$$

\section{ZDENĚK FROLfK}

\begin{abstract}
Hyper-Baire sets and hyper-cozero sets in a uniform space are introduced, and it is shown that for metric-fine spaces the property "every hypercozero set is a cozero set" is equivalent to several much stronger properties like being locally $e$-fine (defined in $\$ 1$ ), or having locally determined precompact part (introduced in $\$ 2$ ). The metric-fine spaces with these additional properties form a coreflective subcategory of uniform spaces; the coreflection is explicitly described. The theory is applied to measurable uniform spaces. It is shown that measurable spaces with the additional properties mentioned above are coreflective and the coreflection is explicitly described. The two coreflections are not metrically determined.
\end{abstract}

This paper was written for the topological conference in Athens, Ohio, October 1972. The results of $\$ 5$ were presented at the International Topological Symposium in Budva, Yugoslavia, August 1972 [F2] (where one can find applications to nonseparable descriptive theory).

At the request of the referee the following is added: This is the third paper in a sequence which is to develop the ideas in [F1] (enriched with the definition of metric-fine due to $A$. Hager [1]) and [F2]. The program is to develop nonseparable descriptive theory (in uniform spaces) by the technique of uniform spaces (in metrizable spaces a lot has been done by R. Hansell [1], [2]). This technique can also be applied to measure theory, see the present author's notes: Mesures uniformes, C. R. Acad. Sci. Paris 277, A105-1108, and Représentation de Riesz des mesures uniformes, ibid. A163-A166. Some further ideas about descriptive theory are in the present author's Basic refinements of uniform spaces, to appear in Proc. 2nd Pittsburgh Topological Symposium, December 1972. "Measurable" hyperextensions of $\sigma$-algebras are studied in [F3].

In general the notation and terminology in E. Čech are used throughout. By a function we mean a mapping into the reals. The set of all uniformly continuous or continuous functions on $X$ is denoted by $U(X)$ or $C(X)$. The bounded functions in $U(X)$ or $C(X)$ are denoted by $U^{*}(X)$ or $C^{*}(X)$.

1. Locally fine spaces. Denote by $p$ or $e$ the reflection of uniform spaces into respectively precompact or separable ones. Thus if $X$ is a uniform space then

Received by the editors March 5, 1973 and, in revised form, November 15, 1973. AMS (MOS) subject classifications (1970). Primary 54E15. 
$p X$ or $e X$ has all finite or countable uniform covers of $X$ for a basis of uniform covers.

Definition 1. A uniform space $X$ will be called respectively locally fine or locally $e$-fine or locally $p$-fine if the cover

$$
\left\{U_{a} \cap V_{a b} \mid a \in A, b \in B\right\}
$$

is uniform if $\left\{U_{a} \mid a \in A\right\}$ is a uniform cover, and if for each $a$ in $A$ respectively, $\left\{V_{a b} \mid b \in B_{a}\right\}$ is a uniform cover of $X$, or of $c X$ or of $p X$.

Definition 2. A uniform space is called $e$-locally fine if the cover (*) is uniform whenever $\left\{U_{a}\right\}$ is a uniform cover of $e X$, and $\left\{V_{a b} \mid b \in B_{a}\right\}$ are uniform covers of $X$.

It should be remarked that there is a general pattern of the two definitions: If $\Phi$ and $\Psi$ are two functors preserving the underlying set, we can define $\Phi$-locally $\Psi$-fine spaces by requiring (*) to be uniform whenever $\left\{U_{a}\right\}$ is a uniform cover of $\Phi X$ and all $\left\{V_{a b}\right\}$ are uniform covers of $\Psi X$.

One can show that the three classes introduced in Definition 1 as well as the class in Definition 2 are coreflective; in subsequent papers the coreflections will be denoted accordingly by $\lambda$ (following Isbell [1]), $\lambda_{e}, \lambda_{p}$ or $\lambda^{e}$. The general pattern is $\lambda_{\psi}^{\phi}$.

Proposition 1. Let $\left\{f_{c} \mid c \in C\right\}$ be a family of uniformly continuous mappings of $X$ into an additive topological group $B$, and let the family $\left\{W_{c}=E\left\{x \mid f_{c} x \neq 0\right\}\right\}$ be uniformly locally finite, and let $f$ be the sum of $\left\{f_{c}\right\}$.

Then eacb of the following conditions guarantees that $f$ is uniformly continuous:

(A) $B=R$ (the reals), $f_{c}$ are bounded functions, and $X$ is locally p-fine.

(B) $B$ is separable, and $X$ is locally e fine.

(C) $X$ is locally fine.

Proof. Let $\left\{U_{a}\right\}$ be a uniform cover of $X$ such that each $U_{a}$ meets a finite number of the sets $W_{a}$ only. The restriction of $f$ to each $U_{a}$ is uniformly continuous as a finite sum of uniformly continuous functions. If $\mathcal{U}$ is a uniform cover of $B$, then $f^{-1}[\mathrm{U}]$ is refined by all $U_{a} \cap f^{-1}[U], U \in \mathcal{U}$. And this cover is uniform under our assumptions (in Case $A$ one may assume that $U$ is finite on each bounded set; in Case B one may assume that $U$ is countable).

We are interested here in the above properties just in connection with measurable spaces. The preceding proposition is included to provide an example of the power of the notions.

2. Uniform spaces with locally determined precompact part. If $X$ is a uniform space we denote by $K(X)$ the Samual compactification of $X$, i.e. the comple- 
tion of $p X$. We assume that $X$ is a subset of $K(X)$. For any uniform cover $\mathcal{U}$ of $X$ we denote by $K(\mathcal{U})$ the union of the closures taken in $K$ of elements of $\mathcal{U}$.

Thus if $X$ is a precompact space than $K(\mathcal{U})=K(X)$ for any uniform cover.

If $V$ is a star-refinement (or only a uniform shrinking) of $\mathcal{U}$ then $K(\mathcal{O}) C$ int $K(\mathcal{U})$.

It should be noted that $f \in U^{*}(X)$ if and only if $f$ is a restriction of a continuous function on $K(X)$.

Remark. Notice that $\bigcap\{K(\mathcal{U})\}$ is just the completion of $X$ (with the uniformity uniquely determined-the traces of $K(U)$ form a basis for uniform covers). Here we are not interested in completeness.

Theorem 1. The following properties of a uniform space $X$ are equivalent:

(1) If $f$ is a bounded function on $X$ such that the restriction of $f$ to eacb element of a uniform cover of $X$ is uniformly continuous, then $f$ is uniformly continuous.

(2) If $f$ is a continuous bounded function on some $K(\mathcal{U}), \mathcal{U}$ being a uniform cover of $X$, then $f$ extends to a continuous function on $K$; i.e. $K=\beta K(\mathcal{U})$ for every uniform cover $U$ of $X$.

(3) $U^{*}(X)=\operatorname{inj} \lim \left\{C^{*}(K(\mathcal{U}))\right\}$.

Proof. Of course $\beta$ in Condition 2 stands for Čech-Stone compactification. The inductive limit in Condition 3 is trivial; since extension is uniquely determined, and since every $f$ in $U^{*}(X)$ extends to $K$, the formula says that $U^{*}(X)$ consists just of restriction of functions in $C^{*}(K(\mathcal{U}))$ for some $\mathcal{U}$. Now it is clear that Conditions 2 and 3 are equivalent.

Assume 1 and prove 2: If $g$ is continuous on $K(U)$ then the restriction $f$ of $g$ to $X$ is uniformly continuous on each $U$ in $U$; indeed, $g$ is continuous on the closure $\bar{U}$ of $U$, and since $\bar{U}$ is compact, $g$ is uniformly continuous with respect to the only uniformity inducing the topology of $\bar{U}$, and hence $g$ is uniformly continuous on the subspace $\bar{U}$ of $K(X)$, hence on the subspace $U$ of $K(X)$, hence on the subspace $U$ of $X$. So if $g$ is bounded, then $f$ satisfies the assumption of Condition 1 , and hence it is uniformly continuous, and hence $f$ extends to a continuous function on $K(X)$, giving a continuous extension of $g$.

Now assume Condition 2, and let $f$ satisfy the assumptions of Condition 1. For any $U, f$ extends to the closure of $U$ in $K(X)$ (because $\bar{U}$ is Samual compactification of the subspace $U$ of $X$ ), and it is easily checked that we get a continuous extension $g$ of $f$ to int $K(\mathcal{U})$; take a $\mathcal{O}$ such that $K(\mathcal{O}) \subset$ int $K(\mathcal{U})$, and apply Condition 2 to the restriction of $g$ to $K(\bigcup)$; now Condition 2 gives us a continuous extension of $g$ to $K$, which is a continuous extension of $f$; thus $f$ is uniformly continuous. 
Proposition 2. Locally pofine spaces enjoy the properties in Theorem 1, and the properties in Theorem 1 imply the following property:

If $\left\{f_{a}\right\}$ is a family of nonnegative uniformly continuous functions sucb tbat the family $\left\{\operatorname{coz} f_{a}\right\}$ is uniformly locally finite and $\left.f=\Sigma f_{a}\right\}$ is bounded, then $f$ is uniformly continuous.

Proof is routine and left to the reader (see Proposition 1).

The properties in Theorem 1 seem to be interesting, and so we shall prove that the spaces with these properties (which may be called by the title of this paragraph) form a coreflective category.

Theorem 2. The class of spaces with the properties in Theorem 1 is coreflective.

Proof. It is enough to show that the class is closed under taking sums and quotients.

Let $X$ be the sum of a family $\left\{x_{a}\right\}$. Then $K(X)$ is the Cech-Stone compactification of the topological sum of $\left\{K\left(X_{a}\right)\right\}$, and one checks easily that if all $X_{a}$ belong to the class then so does $X$.

Let $f: X \rightarrow Y$ be a quotient in uniform spaces and let $\phi: K(X) \rightarrow K(Y)$ be the uniform extension of $f$. If $\mathcal{U}$ is a uniform cover $Y$, then

$$
\phi\left[K\left(X, f^{-1}[\mathcal{U}]\right)\right] \subset K(Y, \text { ใ1). }
$$

Now if $g$ is a bounded continuous function, then the restriction of $g \circ \phi$ to $K(Y, \mathcal{U})$ is continuous, and if $X$ is in our class, then the restriction to $X$ is uniformly continuous, and hence $g$ restricted to $Y$ is uniformly continuous, because $b: Y \rightarrow Z$ is uniformly continuous whenever $b \circ f: X \rightarrow Z$ is. This concludes the proof.

Remark. Notice that we needed just that $f: p X \rightarrow p Y$ is a quotient mapping and $f: X \rightarrow Y$ is uniformly continuous.

3. Uniform hyperextensions of $\mathrm{coz}$ and $B a$. The weakest property in Proposition 2 implies that the uniformly discrete unions of cozero sets are cozero sets. (See the proof of Theorem 7 for an example.) Indeed, if $\left\{B_{a} \mid a \in A\right\}$ is a uniformly discrete family of cozero sets, then we can take uniformly continuous functions $f_{a}$ such that

$$
B_{a}=\operatorname{coz} f_{a}, \quad 0 \leq f_{a} \leq 1,
$$

and then

$$
\bigcup\left\{B_{a}\right\}=\operatorname{coz} \sum\left\{f_{a}\right\}
$$


Definition. For any uniform space $X$ let $b_{X} \operatorname{coz} X$ be the smallest collection of subsets of $X$ which contains $\operatorname{coz} X$ and which is closed under taking of uniformly $\sigma$-discrete unions. The elements of $b \operatorname{coz} X$ are called the hyper-cozero sets in $X$.

Similarly we define hyper-Baire sets in $X$ as follows.

Definition. For any uniform space $X$ let $b_{X} B a X$ denote the smallest $\sigma$ algebra which contains $\operatorname{coz} X$, and which is closed under the operation of taking uniformly discrete unions.

Remark. If $X$ is a metrizable space then $b_{X} B a X$ coincides with the extended Borel sets in $X$ introduced and studied by R. Hansell [1], [2]. R. Hansell was primarily interested in descriptive theory in nonseparable metrizable spaces. We shall study descriptive theory in uniform spaces in a subsequent paper. The reader should be warned not to confuse this hyper-extension of Baire sets with the purely "measurable" hyper-extensions introduced in Frolik [3].

Lemma 1. Let $\pi$ be a finitely multiplicative collection of subsets of a uniform space $X$, and let $\pi$ be the smallest collection wbicb contains $\pi$, and wbicb is closed under the operation of taking the unions of uniformly $\sigma$-discrete families. Then $\mathcal{U}$ is finitely multiplicative.

Proof. Let $R \supset$ be a maximal finitely multiplicative subcollection of $\mathbb{N}$. Let $\mathcal{S}$ consist of the unions of uniformly $\sigma$-discrete families ranging in $\tilde{G}$. We shall show that $\delta$ is finitely multiplicative; then $\delta=R$, hence $R=\pi$.

Let $M_{i}=\bigcup\left\{M_{a}^{i n} \mid n \in N, a \in A_{n}^{i}\right\}$ for $i=1$, 2, where $\left\{M_{a}^{i n} \mid a \in A_{n}^{i}\right\}$ are uniformly discrete families in $R$. Then $M_{1} \cap M_{2}$ is the union of all $M_{a}^{1 n} \cap M_{b}^{2 m}$, and the family is uniformly $\sigma$-discrete, because each family

$$
\left\{M_{a}^{1 n} \cap M_{b}^{2 m} \mid a \in A_{n^{\prime}}^{1} b \in A_{m}^{2}\right\}
$$

is uniformly discrete.

Corollary. $b_{X} \operatorname{coz} X$ is finitely multiplicative.

It should be remarked that if $\operatorname{coz} X$ is countably multiplicative then $b_{X} \operatorname{coz} X$ is not in general.

Lemma 2. Let $X$ be a uniform space. Denote by $\mathcal{S}$ the set of all mappings of $X$ into separable metric spaces sucb that the preimages of open sets are bypercozero sets in $X$. Denote by $\mathcal{U}$ all maps into the reals whicb belong to $\delta$, and let $\mathrm{U}^{*}$ be the set of all bounded functions in $\mathcal{U}$. Then:

(a) If $f_{i}: X \rightarrow S_{i}$ belong to $S_{,}$then so does the reduced product $\left.f: X \rightarrow \mathbb{I} S_{i}\right\}$ of $\left\{f_{i}\right\}$.

(b) If $f: X \rightarrow S$ belongs to $S$, and if $g$ is a continuous mapping of $S$ into a. 
separable metric space $T$, then $g \circ f: X \rightarrow T$ belongs to $S$.

(c) $\mathcal{U}$ is closed in the topology of uniform convergence.

(d) $\mathcal{U}$ is closed under the operation of taking the sums of uniformly discrete families.

(e) $\mathrm{U}^{*}$ is closed under the operation of taking the sums of uniformly discrete families of equibounded functions.

(f) $\mathcal{U}$ is an inversion-closed algebra.

(g) $b_{X} \operatorname{coz} X=\operatorname{coz} U^{*}$ ( $=$ the set of all $\operatorname{coz} f, f \in \mathcal{U}^{*}$ ).

(h) The collection of all countable covers of $X$ by elements of $b_{X} \operatorname{coz} X$ is a basis for a uniformity (wbich is necessarily metric-fine). The point finite covers also form a basis.

Proof. Assertion (a) follows from Lemma 1. Indeed, if $U_{i}$ are open in $S_{i}$ for $i=1, \ldots, n$ then the obvious relation

$$
\bigcap\left\{f_{i}^{-1}\left[U_{i}\right]=\left\{x \mid f_{i} x \in U_{i}\right\}\right.
$$

shows that the preimages of canonical open sets in the product are hyper-Baire sets. Assertion (b) is obvious. Assertion (c) follows from (a) and (b): If $\left\{f_{n}\right\}$ in $\mathcal{U}$ uniformly converges to $f: X \rightarrow R$, then by (a) there exists a surjective mapping $g: X \rightarrow S \in \mathcal{S}$, and continuous functions $g_{n}: S \rightarrow R$ such that $f_{n}=g_{n} \circ g$ (take the image of the reduced product of $\left\{f_{n}\right\}$ for $S$ ); then $\left\{g_{n}\right\}$ converges uniformly to a continuous function $b: S \rightarrow R$, and clearly $f=b \circ g$. Hence $f$ belongs to $\mathcal{U}$ by Assertion (b). Assertions (d), (e) and (f) are evident.

To prove Assertion ( $\mathrm{g}$ ), let $\mathbb{B}$ be the collection of the preimages of open sets under mappings from $\mathcal{S}$; by Assertion 2

$$
B=\operatorname{coz} \mathcal{U}^{*} \cdot
$$

We shall prove that $B=b_{X} \operatorname{coz} X$; it suffices to show that $B$ is closed under countable unions (and this follows from Assertions (c) and (f)), and uniformly discrete unions, and this follows from Assertion (e).

Finally, to prove the last assertion we must show that each countable cover $\left\{U_{n}\right\}$ in Assertion $(\mathrm{h})$ has a star-refinement of the same type. By $(\mathrm{g})$ we can choose $f_{n}$ in $\mathcal{U}$ such that $U_{n}=\operatorname{coz} f_{n}$. By (a) the reduced product $f$ of $\left\{f_{n}\right\}$ is in $\mathcal{S}$, hence $f: X \rightarrow f[X]$ is in $\mathcal{S}$. Now $U_{n}=f^{-1}\left[V_{n}\right]$ where $V_{n}$ is open in $f[X]$. Take an open star-refinement $\left\{W_{i}\right\}$ of $\left\{n_{i}^{n}\right\}$, clearly $\left\{f^{-1}\left[W_{i}\right]\right\}^{n}$ is the required starrefinement of $\left\{U_{n}\right\}$. We can take $\left\{W_{i}\right\}$ to be locally finite, hence point-finite. This concludes the proof. The parenthesized statement will be explained in the next paragraph. 
Proposition 3. The byper-Baire sets in $X$ form the smallest collection which contains the cozero sets, and which is closed under countable intersections, and uniformly $\sigma \cdot d$ iscrete unions.

Proof is left to the reader. It shows that the complemented part of the collection in Proposition 3 is closed under the same operations.

4. Metric-fine locally $e$-fine spaces. It is proved that for metric-fine spaces all the properties in paragraphs 2 and 3 coincide, and a coreflection into metricfine spaces which enjoy the properties will be described.

If $X$ is a uniform space, we denote by $a X$ the set $X$ endowed with the finest uniformity compatible with uniform topology. Now $X$ is called metric-fine if for every uniform map $f$ of $X$ into a metric space $Y$ the map $f: X \rightarrow \alpha Y$ is uniformly continuous. If this requirement restricts to separable metric $Y$ 's, then one gets the definition of (separable metric)-fine spaces. Recall several results from [F2], [F4].

The metric-fine spaces are coreflective in uniform spaces, and a coreflection of $X$, denoted by $m X$, is the set $X$ endowed with the uniformity which has for a basis of uniform covers the uniformly $\sigma$-discrete (in $X !$ ) covers of $X$ which are completely $\operatorname{coz} X$-additive.

The (separable metric)-fine spaces are also coreflective and if the coreflection of $X$ is denoted by $m_{K_{0}} X$, then $e m X=e m_{K_{0}} X$, and this uniformity has for a basis of uniform covers all countable covers of $X$ by cozero sets in $X$. It is quite easy to check that $m X=e_{m_{x_{0}}} X$.

The orem 3. The following conditions on a metric-fine space $X$ are equivalent:

(1) $X$ is locally e-fine.

(2) $X$ is locally p-fine.

(3) $X$ enjoys the properties in Theorem 1.

(4) $X$ enjoys the weakest property in Proposition 2.

(5) $b_{X} \operatorname{coz} X=\operatorname{coz} X$, i.e. every byper-cozero set is a cozero set.

Proof. Self-evidently (1) implies (2). By Proposition 2, Condition (2) implies (3), and (3) implies (4). By the introduction to $\$ 2$, Condition (4) implies (5). Thus without any additional properties of $X$, the conditions are weaker and weaker. We show that if $X$ is metric-fine then (5) implies (1).

A typical uniform cover of $X$ is of the form $\left\{U_{a}^{n} \mid a \in A_{n}, n \in N\right\}$ where each $\left\{U_{a}^{n} \mid a \in A_{n}\right\}$ is a uniformly discrete family, and its union $B_{n}^{n}$ is a cozero set in $X$ (this is equivalent to the description given above). Let

$$
U_{a}^{n}=\bigcup\left\{V_{a}^{n k} \mid k \in N\right\}
$$

where $V_{a}^{n k}$ are cozero sets in $X$. We must show that 


$$
\left\{V_{k}^{a n} \mid a \in A_{n}, n \in N, k \in N\right\}
$$

is a uniform cover of $X$. For each $n$ and $k$ let $C_{n k}=\bigcup\left\{V_{a}^{n k} \mid a \in A_{n}\right\}_{0}$. By Condition (5) the sets $C_{n k}$ are cozero sets in $X$, and hence the cover $\left\{V_{a}^{n k}\right\}$ is uniform because $X$ is metric-fine. This concludes the proof.

Remark. Further study of the conditions in Theorem 3 may be of interest.

Theorem 4. For each uniform space $X$ let $\underline{\mu} X$ be the set $X$ equipped with the uniformity which has for a basis of uniform covers the uniformly $\sigma$-discrete covers ranging in $b_{x} \operatorname{coz} X$. Then

(a) $e \underline{\mu} X$ bas for a basis of uniform covers all the countable covers ranging in $b_{X} \operatorname{coz} X$.

(b) $\operatorname{coz} \underline{\mu} X=b_{X} \operatorname{coz} X$.

(c) $\underline{\mu}$ is a coreflection into locally e-fine metric-fine spaces.

Proof. First we must show that the defining covers form a basis. The meet of two such covers is another, by the corollary to Lemma 1 . So we must show that every defining cover is star refined by a defining cover. Let $\left\{U_{a}^{n} \mid n \in N, a \in A_{n}\right\}$ be a defining cover; certainly we assume that $\left\{U_{a}^{n} \mid a \in A_{n}\right\}$ is uniformly discrete, and we denote by $B_{n}$ the union. Thus $\left\{B_{n}\right\}$ is a countable cover ranging in $b_{X} \operatorname{coz} X$. By Lemma 2, we can take a point-finite cover $\left\{B_{n}^{\prime}\right\}$ ranging in $b_{X} \operatorname{coz} X$ such that $B_{n}^{\prime} \subset B_{n}$; then the cover $\left\{U_{a}^{n} \cap B_{n}^{\prime}\right\}$ is defining, and refines the given cover. Thus we may and shall assume that $\left\{B_{n}\right\}$ is point-finite. Again by Lemma 2 we can take a countable cover $\left\{C_{k}\right\}$ ranging in $b_{X} \operatorname{coz} X$, which star refines $\left\{B_{n}\right\}$. Let $\mathcal{U}_{k}$ be the family of all sets

$$
C_{k} \cap U_{a}^{n} \cap \cap \cdots \cap U_{a}^{n} m
$$

where $n_{i}$ are all integers $l$ such that $C_{k} \subset B_{l}$. Clearly each family $\mathcal{U}_{k}$ is uniformly discrete and ranges in $l_{X} \operatorname{coz} X$. It is easy to check that $U\left\{U_{k}^{k}\right\}$ is a starrefinement of the given cover.

Proof of (a). Every countable cover by hyper-Baire sets is a uniform cover of $\underline{\mu} X$, and hence it is a uniform cover of $e^{\mu} X$. Conversely assume that $\left\{W_{k}\right\}$ is a countable uniform cover of $\mu X$; we must find a countable cover $\left\{B_{k}\right\}$ which rang* es in the hyper-cozero sets, and which refines $\left\{w_{k}\right\}$. Choose a defining cover $\left\{U_{a}^{n}\right\}$ and put

$$
B_{k}=\bigcup\left\{U_{a}^{n} \mid U_{a}^{n} \subset w_{k}\right\}
$$

It is easy to check that $\left\{B_{k}\right\}$ is a cover with the properties required.

Proof of (b). This is an immediate consequence of statement (a). Indeed, if 
$f: \mu X \rightarrow S$, is uniformly continuous, $S$ being separable metric, then $f: e \underline{X} \rightarrow S$ is uniformly continuous, and if $B_{n}$ is a countable cover of $X$ ranging in hyper-cozero sets in $X$ such that the diameter of each $f[B], B \in B_{n}$, is at most $1 / n$, then for every open set $U$ in $S$ we have

$$
f^{-1}[U]=\bigcup\left\{B \mid B \subset U, B \in \bigcup \mathscr{B}_{n}\right\}
$$

and hence $f^{-1}[U]$ is a hyper-cozero set.

Proof of $(\mathrm{c})$. The space $\underline{\mu} X$ is metric-fine because every cover $\left\{B_{n} \cap U_{a}^{n} \mid\right.$ $\left.n \in N, a \in A_{n}\right\}$ where $\left\{U_{a}^{n} \mid a \in A_{n}\right\}$ is a uniform cover of $\underline{\mu} X$, and $B_{n} \in \operatorname{coz} \underline{\mu} X$, has a uniformly $\sigma$-discrete refinement by sets in $b_{X} \operatorname{coz} X=\operatorname{coz} \underline{\mu} X$, and hence it is a uniform cover of $\underline{\mu} X$. Thus all the conditions in the Theorem are equivalent, and it is enough to show that $\operatorname{coz} \underline{\mu} X$ is closed under uniformly discrete (in $\underline{\mu} X)$ unions. But if $B$ is the union of a uniformly discrete (in $\underline{\mu} X)$ family $\left\{B_{c} \mid c \in C\right\}$ then we write $B=\bigcup\left\{B_{n} \mid n \in N\right\}$ such that $\left\{B_{n^{\prime}} \cap B_{c} \mid c \in C\right\}$ is uniformly discrete in $X$, and $B_{n}$ 's are in $\operatorname{coz} \underline{\mu} X$. Indeed, if $\left\{U_{a}^{k}\right\}$ is a defining cover of $\underline{\mu} X$ such that each $U_{a}^{n}$ meets one $B_{c}$ at most, then the union $U_{n}$ of all $U_{a}^{n}$, with $n$ fixed, has the property that $\left\{B_{c} \cap U_{n} \mid c \in C\right\}$ is discrete in $X$. This concludes the proof of the fact that $\underline{\mu} X$ is in our class. Now let $f$ be a uniformly continuous mapping of a locally $e$-fine metric-fine space $Y$ into $X$; we must show that $f: Y \rightarrow \underline{\mu} X$ is uniformly continuous. Since $\left\{Z \rightarrow b_{Z} \operatorname{coz} Z\right\}$ is functorial, the preimages of hyper-cozero sets under $f$ are hyper-cozero sets in $Y$, hence they are cozero sets in $Y$ by Theorem 3. Now if $\left\{U_{a}^{n}\right\}$ is a defining cover for $\mu X$, then the preimage of

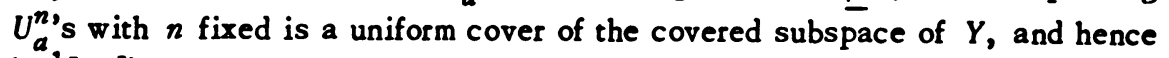
$\left\{f^{-1}\left[U_{a}^{n}\right]\right\}$ is a uniform cover of $Y$ because $Y$ is metric-fine. This concludes the proof.

5. Locally e-fine measurable spaces. Measurable spaces were introduced in Frolik [2] as uniform spaces $X$ such that the pointwise limit of any sequence of uniformly continuous mappings into a metrizable space is uniformly continuous. It is shown that these spaces are coreflective in uniform spaces, and the coreflection $M X$ of $X$ has for a basis of uniform covers the uniformly $\sigma$-discrete partitions of $X$ of bounded class in BaX; equivalently, the covers of the form

$$
\left\{B_{n} \cap U_{a}^{n} \mid n \in N, a \in A_{n}\right\},
$$

where $\left\{B_{n}\right\}$ is a partition of $X$ into Baire sets, and $\left\{B_{n} \cap U_{a}^{n} \mid a \in A_{n}\right\}$ is uniformly discrete in $X$.

Thus $X$ is measurable if and only if $\operatorname{coz} X=B a X$, and $X$ is metric-fine.

Since every measurable space is metric-fine, the next result is an immediate consequence of Theorem 3. 
Theorem 5. For a measurable space the conditions in Theorem 3 are equivalent.

Theorem 6. For each uniform space $X$ let $\bar{\mu} X$ be the set $X$ endowed with the uniformity which bas all uniformly $\sigma$-discrete (in $X$ ) partitions of $X$ into byperBaire sets in $X$ for a basis of uniform covers. Then:

(a) $e \bar{\mu} X$ bas for a basis of uniform covers the countable partitions of $X$, which consist of byper-Baire sets in $X$.

(b) $\operatorname{coz} \bar{\mu} X=B a \bar{\mu} X=b_{X} B a X$.

(c) $\bar{\mu} X$ is a coreflection of $X$ in locally e-fine measurable space.

Proof. It is obvious that the covers in question really form a basis for a uniformity (this required a proof in Theorem 4). The remainder follows the pattern of the proof of Theorem 4. The details are left to the reader.

A coreflection $\phi$ of uniform spaces is called metrically determined if for each uniform space $X$, the space $\Phi X$ is projectively generated by all maps $\phi f$ where $f: X \rightarrow Y$ is uniformly continuous with $Y$ metrizable.

Theorem 7. The functors $\underline{\mu}$ and $\bar{\mu}$ are not metrically determined. If $Z$ is an uncountable uniformly discrete space, and if $X$ is the product of an uncountable number of copies of $Z$, then neither $\underline{\mu} X$ nor $\bar{\mu} X$ is metrically determined.

Proof. It will be obvious that it is enough to consider the case $X=\Pi\left\{M_{\alpha} d\right.$ $\left.\alpha<\omega_{1}\right\}$ where $M_{a}=M$ for each $\alpha$, and $M$ is an uncountable set. Choose an injection $f$ of the countable ordinals into $M$. The following partition is a uniform cover of $\underline{\mu} X$, hence of $\bar{\mu} X$. For $m$ in $M$ let

$$
\begin{aligned}
U_{m} & =E\left\{x \mid x_{0}=m\right\} \text { if } m \notin E f, \\
& =E\left\{x \mid x_{0}=m, x_{a}=m \text { where } m=f_{a}\right\} \text { otherwise. } \\
V_{m} & =\phi \text { if } m \notin E f, \\
& =E\left\{x \mid x_{0}=m, x_{a} \neq m \text { where } m=f_{a}\right\} \text { otherwise. }
\end{aligned}
$$

The partition $\left\{E\left\{x \mid x_{0}=m\right\}, m \in M\right\}$ is a uniform cover of $X$, and the partition $\left\{U_{m}\right\}$ $u\left\{V_{m}\right\}$ is obtained just by splitting some of the members into two cozero sets in $X$. If $b$ is any uniformly continuous mapping of $X$ into a metric space $S$, then $b$ factorizes through a projection of $X$ onto a countable subproduct, and hence $\left\{b^{-1}[(S)] \mid s \in S\right\}$ does not refine our partition, because our partition depends on an uncountable number (in fact all) coordinates. Hence neither $\underline{\mu} X$ nor $\bar{\mu} X$ is metrically determined.

At the request of the referee we add the following theorem from [F2]:

If $X$ is a metric space which is completely metrizable (or, more generally, 
Souslin in some completely metrizable space) then

$$
\text { bi-Souslin } X=\text { hyper (Baire } X)=\text { hyper-Baire }(X)=\operatorname{coz}(\bar{\mu} X)
$$

where bi-Souslin $X$ stands for all $Y \subset X$ such that both $Y$ and $X-Y$ are Souslin sets in the topological space $X$, hyper (Baire $X$ ) is the measurable hyper-extension from [F3], and hyper-Baire $(X)$ and $\bar{\mu} X$ are defined above.

\section{REFERENCES}

E. Čech, Topological spaces, 2nd ed., Publ. House Czech Acad. Sci., Prague, 1966; English transl., Wiley, New York, 1966. MR 35 \#2254.

Z. Frolík,

[F1] Topological methods in measure theory and the theory of measurable spaces, General Topology and its Relations to Modem Analysis and Algebra (Proc. 3rd Prague Sympos., 1971), Academia, Prague, 1972, pp. 127-139.

[F2] Interplay of measurable and uniform spaces, Topology and its Applications (Proc. 2nd Yugoslavia Sympos., Budva, 1972), Beograd, 1973, pp. 96-99.

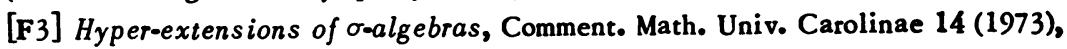
361-375.

[F4] A note on metric-fine spaces, Proc. Amer. Math. Soc. (to appear).

[F5] Measurable uniform spaces, Pacific J. Math. (to appear),

A. Hager,

[1] Some nearly fine uniform spaces, Proc. London Math. Soc. (to appear).

[2] Measurable uniform spaces, Fund. Math. 77 (1972), 51-73.

R. Hansell,

[1] Thesis, Rochester, 1970.

[2] On the nonseparable theory of Borel and Souslin sets, Bull. Amer. Math. Soc. 78 (1972), 236-241. MR $45 \# 3211$.

J. Isbell, Uniform spaces, Math. Surveys, no. 12, Amer. Math. Soc., Providence, R. Io, 1964. MR 30 \#561

MATEMATICKY USTAV, ČSAV, PRAGUE, CZECHOSLOVAKIA

DEPARTMENT OF MATHEMATICS, UNIVERSITY OF PITTSBURGH, PITTSBURGH, PENNSYLVANIA 15213 